\title{
Suivi des évolutions topographiques en environnement littoral ou fluvial par imagerie de résistivité : étude en modèle réduit
}

\author{
R. Maupin ${ }^{(1)}$, S. Palma Lopes ${ }^{(2)}$, I. Papin ${ }^{(1)}$, J. Alexandre ${ }^{(2)}$ \\ ${ }^{1}$ Géomarine, 4, av. du 8 mai 1945, 17630 La Flotte- \\ rmaupin@nordnet.fr \\ ${ }^{2}$ Laboratoire Central des Ponts et Chaussées, route de Bouaye, \\ BP 4129,44341Bouguenais CEDEX-sergio.lopes@lcpc.fr
}

\section{Résumé}

Les techniques de relevé topographique en environnement littoral ne permettent pas aisément un suivi continu des mouvements sédimentaires. Géomarine et le LCPC ont proposé une méthode, issue des techniques de prospection électrique à courant continu, et étudient sa faisabilité. Cet article présente les résultats d'une modélisation physique à échelle réduite en cuve, tire les premières estimations de sensibilité, et conclut sur les essais et développements encore nécessaires pour valider la méthode.

\begin{abstract}
Topographic survey techniques in coastal environments do not provide easy means of following up sedimentary evolutions. Géomarine and the LCPC suggested a method adapted from the direct current geoelectrical prospecting techniques, and are examining its feasibility. In this paper, we present reduced scale model experimentations in a tank, we draw the first estimations of the sensitivity, and we conclude on the tests and developments that still need to be carried out in order to validate the method.
\end{abstract}

\section{Introduction}

Actuellement, les techniques de mesure topographique en zones mobiles ne permettent pas un suivi en continu des évolutions des ensembles sédimentaires. Elles se limitent à des mesures ponctuelles effectuées à un instant donné : l'utilisation du sonar nécessite une hauteur d'eau minimale et une houle faible, et au contraire le tachéomètre s'utilise sur site émergé. Au niveau de zones littorales immergées sous peu d'eau et soumises à une agitation, il y a un manque réel de mesures topographiques. Le suivi en continu de ces sites serait un outil indispensable pour caractériser les évolutions à moyen et court terme : suivi d'un chenal ou d'une flèche sableuse, d'une cote d'envasement ou d'un niveau 
d'ensablement. De nombreuses applications peuvent être envisagées en environnement littoral (plages sableuses, ports) ou fluvial.

Géomarine et le LCPC étudient depuis 1998 l'adaptation d'une méthode issue de la géophysique appliquée (Maupin, 1999) : la méthode de prospection électrique à courant continu en site aquatique (Lagabrielle, 1984 ; Baumgartner, 1996). Cette étude de faisabilité est maintenant inscrite dans les actions du Programme National Environnement Côtier (PNEC), dans le cadre d'une collaboration (BRGM, Géomarine, LCPC, CETMEF).

Après avoir effectué une première approche par modélisations numériques, un modèle physique à petite échelle a été réalisé. Nous en synthétisons ici les résultats.

\section{La méthode proposée}

\subsection{Rappels sur la prospection électrique à courant continu}

Les méthodes de prospection électrique s'intéressent à la résistivité électrique des terrains, qui est leur faculté à résister au passage d'un courant électrique. La résistivité dépend de la nature du sol, de sa porosité, de son degré de saturation et de la minéralisation de l'eau de formation. Elle est de plus fonction de la température.

Quatre électrodes sont nécessaires pour effectuer une mesure (figure 1): deux électrodes $\mathbf{A}$ et $\mathbf{B}$ permettent d'injecter un courant continu dans le terrain, tandis que deux électrodes $\mathbf{M}$ et $\mathbf{N}$ permettent de mesurer une différence de potentiel induite par la circulation de ce courant dans le sol. On en déduit une grandeur appelée résistivité apparente, notée $\rho_{\mathrm{a}}$ et exprimée en Ohm.mètres $(\Omega \mathrm{m})$. La résistivité apparente mesurée n'est pas la résistivité vraie du sous-sol (propriété locale) mais une mesure globale sur un certain volume de terrain. Plus les électrodes sont écartées (longueur du dispositif AMNB) et plus le volume de terrain influant sur la mesure est grand.

Si l'on effectue des mesures en déplaçant latéralement ce dispositif, à écartement constant des électrodes, on obtient un traîné de résistivité apparente. Au contraire si l'on écarte progressivement les électrodes, à milieu de dispositif fixe, on obtient un sondage électrique (rayon d'investigation croissant). Grâce aux systèmes automatisés, on peut combiner ces deux techniques : un câble multiélectrodes est installé et un séquenceur permet de balayer successivement toutes les combinaisons de 4 électrodes que l'on souhaite sur ce câble (figure 1). C'est la technique du panneau électrique.

\subsection{Principe de la méthode}

Il s'agit d'adapter la technique des panneaux électriques. L'innovation consiste à enfouir le câble multiélectrodes sous une épaisseur de quelques mètres de sédiments afin d'imager la topographie de l'interface eau / sédiments (figure 1). La ligne multiélectrodes est reliée en surface à un résistivimètre automatisé. On 
s'attend à ce que des variations de topographie de l'interface influent sur le résultat du panneau électrique. Par exemple, un apport de sédiments (ensablement, flèche sableuse), de résistivité supérieure à celle de l'eau, devrait faire augmenter la résistivité apparente des dispositifs qui intègrent ce volume dans leur mesure. L'orientation de la ligne électrique, l'installation d'une ou plusieurs lignes en parallèle et la profondeur d'enfouissement, dépendent du site d'application et de la problématique.

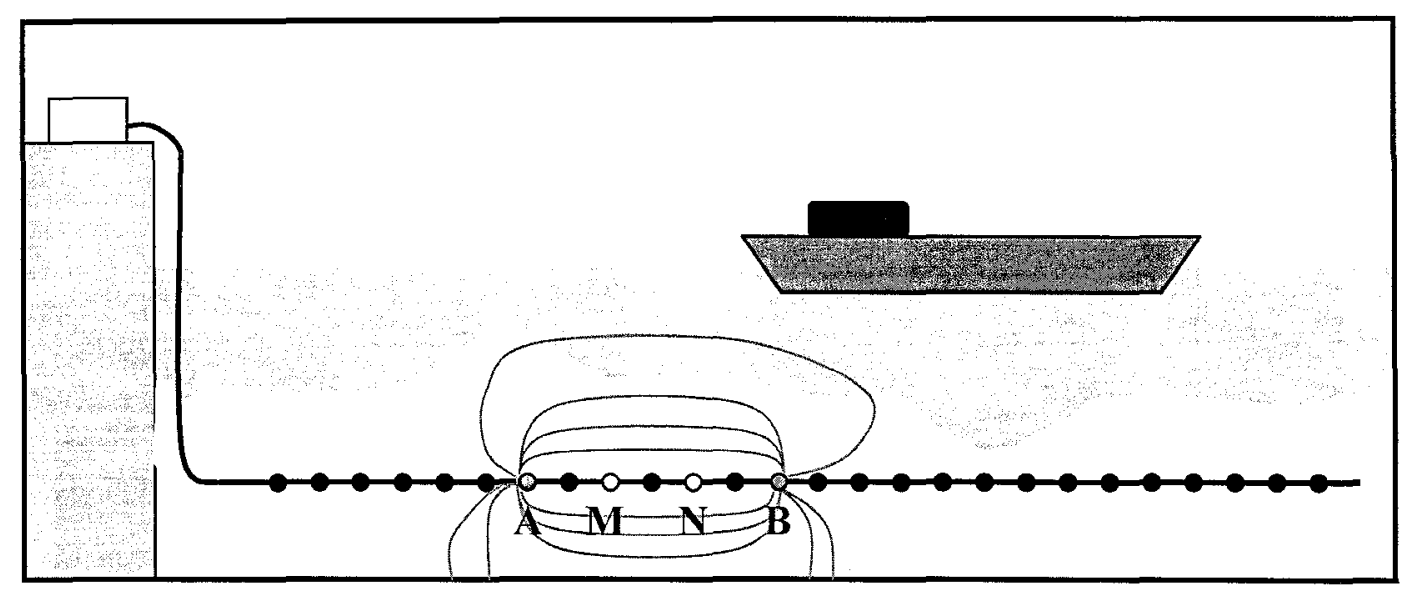

Figure 1. Principe de la méthode (en rouge : lignes de courant).

Principle of the method (in red: current lines).

\section{Dispositif expérimental}

\subsection{Description du modèle physique}

Nous avons travaillé dans une cuve en PVC de longueur $2 \mathrm{~m}$, de largeur 1,20 m et de hauteur $0,80 \mathrm{~m}$ (figure 2).

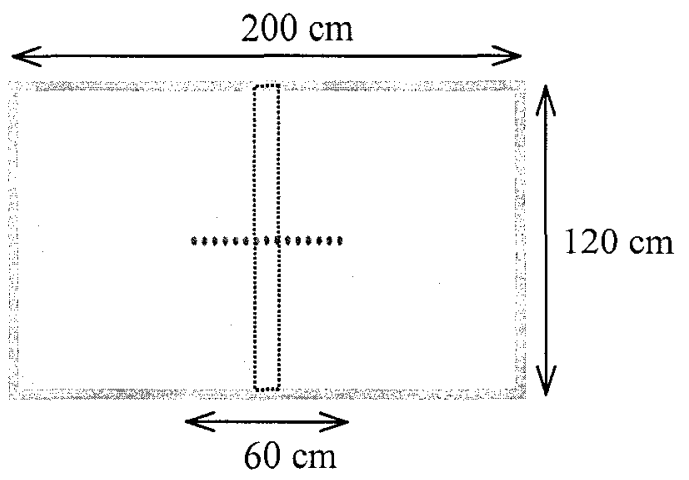

Figure 2. Dimensions du modèle et position des d'électrodes (points rouges).

Model dimensions and position of the electrodes (red dots).

La cuve a été remplie d'une couche sédimentaire sableuse saturée en eau dans laquelle a été installée la ligne multiélectrodes. Après avoir fait quelques pré-tests 
avec de l'eau salée et du sable de plage non trié, il a été décidé de simplifier les paramètres du modèle : le choix du «sédiment» s'est porté sur du sable de Fontainebleau, de granulométrie bien connue (centrée autour de $200 \mu \mathrm{m}$ ), et nous avons préféré l'eau douce dans un premier temps, moins pénalisante pour la méthode et plus facile à obtenir et à contrôler en laboratoire (résistivité, température). Le sédiment a été mis en place par «pluviation» de couches successives dans l'eau pour garantir une saturation et si possible une porosité homogènes, en dehors des zones proches des bords.

\section{2 Électrodes et système d'acquisition}

La ligne multiélectrodes est constituée d'un câble fin multiconducteur sur lequel sont placées 16 électrodes en cuivre espacées de $4 \mathrm{~cm}$. Cette ligne a été posée au centre de la cuve sur une couche de $42 \mathrm{~cm}$ de sable saturé, puis recouverte successivement de couches d'eau et de sable afin de simuler différentes situations de marée et d'enfouissement. Notons que la longueur de la partie «active » du câble n'est que de $60 \mathrm{~cm}$ au centre de la cuve, donc assez loin des bords (figure 2). Le matériel d'acquisition de panneaux électriques est constitué d'un résistivimètre et d'un boîtier de commutation pour effectuer toutes les combinaisons d'électrodes souhaitées, ici des combinaisons de type Wenner (électrodes équidistantes : $\mathbf{A M}=\mathbf{M N}=\mathbf{N B}$ ).

\section{Résultats expérimentaux}

\subsection{Configurations en estran plan}

Pour ces essais nous avons fait varier la profondeur d'enfouissement de la ligne de mesure (épaisseur de sable saturé la recouvrant) et la hauteur d'eau (marée), à interface eau / sédiments plane. L'intérêt est de tester la sensibilité de la méthode pour suivre la cote de l'interface (problématiques de type "érosion» ou « ensablement », à topographie plane). On se trouve ici dans une configuration 1D avec un modèle de terrain tabulaire stratifié horizontalement. On peut alors appliquer la technique du sondage électrique, c'est à dire mesurer les variations de la résistivité apparente en fonction de la longueur du dispositif AMNB.

Sur la figure 3 sont représentés les sondages électriques de 16 situations principales modélisées. Ce sont des courbes de sondage classiques de type « 3 terrains »: à mesure que la longueur de dispositif augmente, les terrains progressivement intégrés à la mesure sont : d'abord le sable saturé (dans lequel les électrodes sont noyées), puis l'eau (moins résistive que le sable), puis l'air d'un côté et le fond de la cuve de l'autre (tous deux infiniment résistifs). Les différentes courbes obtenues sont bien distinctes et chacune est caractéristique d'une situation donnée. 


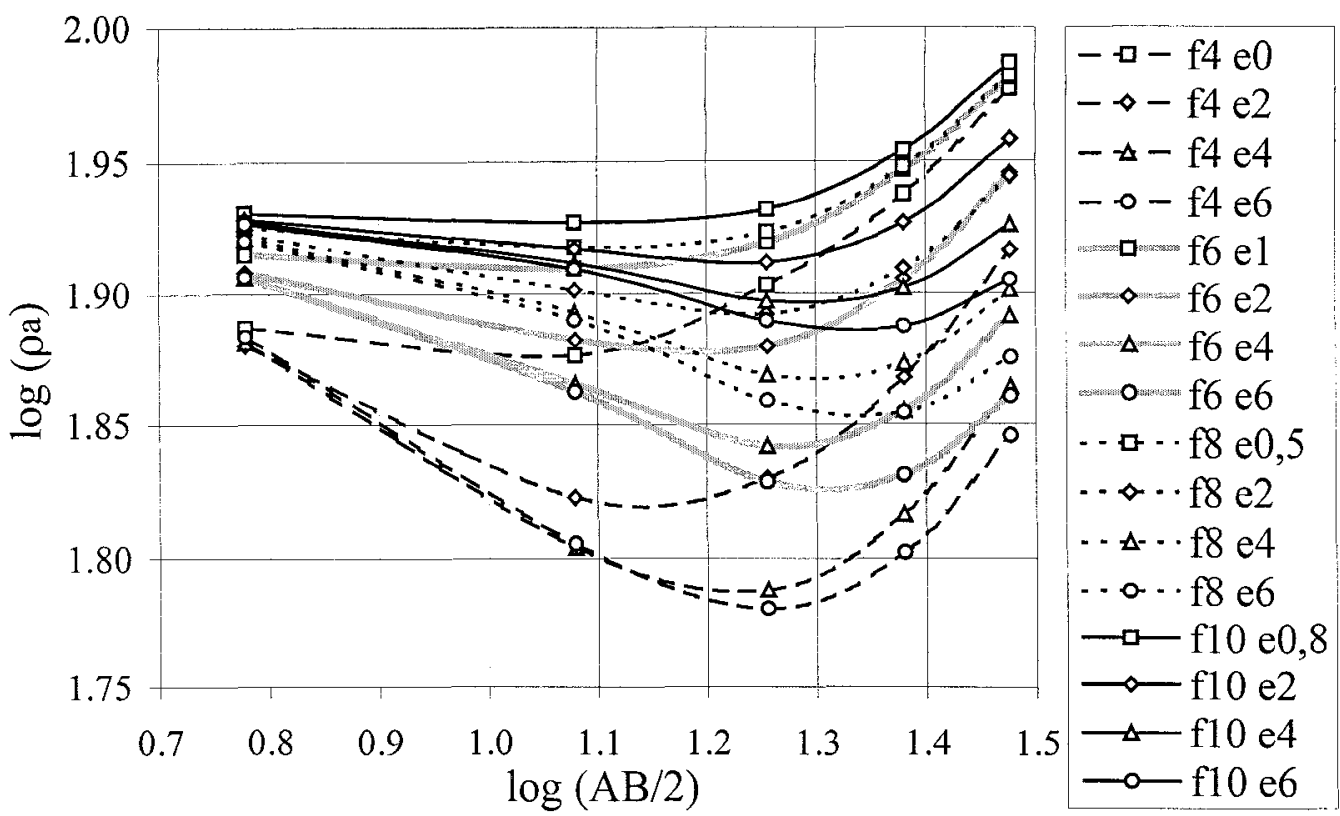

Figure 3. Sondages électriques en fonction de la profondeur d'enfouissement des électrodes (ex. 18 pour $8 \mathrm{~cm}$ ) et de la hauteur d'eau (ex. e4 pour $4 \mathrm{~cm}$ ). Vertical electrical soundings versus electrode burying depth (e.g. $f 8$ means $8 \mathrm{~cm}$ ) and water depth (e.g. e4 means $4 \mathrm{~cm}$ ).

La résolution du problème inverse $1 \mathrm{D}$ est couramment appliquée en prospection électrique à terre ou en site aquatique et il sera aisé de l'adapter à la configuration avec une ligne enfouie. Un tel calcul n'a pas été efficace dans le cadre de notre modèle, pour des raisons liées aux bords de la cuve, inexistants dans la réalité.

En extrapolant les résultats des essais à un cas réel en eau douce, avec une ligne enfouie sous 2 mètres de sédiments et comportant des électrodes espacées de 2 mètres, on peut espérer que la résolution sur la variation de position de l'interface eau / sédiments sera de l'ordre de quelques décimètres.

\subsection{Configurations à topographie variable}

\subsubsection{Morphologies testées}

Des perturbations morphologiques simples ont été réalisées à l'interface eau / sédiments. Ces modifications sont du type «chenal » ou « flèche sableuse » rectilignes, et ont été pratiquées au centre de la cuve, perpendiculairement à la ligne multiélectrodes (pointillés sur la figure 2). Le tableau 4 décrit les essais les plus significatifs. Les dimensions des morphologies présentées sont similaires : ce sont les dimensions aisément détectables dans le modèle réduit. Les morphologies sont de longueur «infinie » perpendiculairement à la ligne (structures 2D, cf. figure 2). 


\begin{tabular}{|c|c|c||c|c|}
\hline \multicolumn{3}{|c||}{ morphologie } & \multicolumn{2}{c|}{ configuration } \\
\hline type & hauteur & largeur & sable & eau \\
\hline chenal & $2 \mathrm{~cm}$ & $5 \mathrm{~cm}, 9 \mathrm{~cm}$ & $4 \mathrm{~cm}$ & $6 \mathrm{~cm}$ \\
\hline flèche & $2 \mathrm{~cm}$ & $12 \mathrm{~cm}, 7 \mathrm{~cm}$ & $4 \mathrm{~cm}$ & $6 \mathrm{~cm}$ \\
\hline chenal & $2 \mathrm{~cm}$ & $5 \mathrm{~cm}, 10 \mathrm{~cm}$ & $10 \mathrm{~cm}$ & $2 \mathrm{~cm}$ \\
\hline flèche & $2 \mathrm{~cm}$ & $10 \mathrm{~cm}, 3 \mathrm{~cm}$ & $10 \mathrm{~cm}$ & $4 \mathrm{~cm}$ \\
\hline
\end{tabular}

Tableau 4. Caractéristiques des essais : dimensions de la morphologie (hauteur, largeurs basse et haute) et configuration (hauteur de sable recouvrant la ligne, hauteur d'eau). Characteristics of the testings : morphology dimensions (height, bottom and top widenesses) and configuration (sand height over the electrode line, water depth).

\subsubsection{Analyse des résultats}

La méthode proposée est essentiellement relative: les panneaux électriques pourront être effectués très régulièrement, et l'on comparera les évolutions entre deux panneaux successifs. Chacun des résultats suivants est donc normalisé, point par point, à la situation juste antérieure, avant réalisation de la morphologie (estran plan). On obtient ainsi des pseudosections relatives où chaque point est un rapport de résistivités apparentes, sans dimension. Par convention, chaque résistivité apparente mesurée avec un dispositif AMNB donné est représentée en un point dont l'abscisse $\mathbf{x}$ est le milieu du dispositif et l'ordonnée est la demi longueur $\mathbf{A B} / 2$ de ce dispositif. Ces pseudosections ne sont pas des coupes de terrain, puisqu'une résistivité apparente est une mesure qui «intègre» les résistivités vraies sur un certain volume. Elles permettent cependant de mettre en évidence, qualitativement, l'effet des morphologies testées.

Dans le cas d'un chenal (figure 5, pseudosections du haut), on a retiré du sable saturé (dont la résistivité vraie est d'environ $80 \Omega \mathrm{m}$ ) dans un certain volume qui est investi par de l'eau (environ $30 \Omega \mathrm{m}$ ). Une partie du milieu qui surplombe la ligne de mesure devient donc plus conducteur : les points de la pseudosection relative où la mesure est affectée par la présence du chenal montrent des valeurs inférieures à 1 . Inversement, dans le cas d'une flèche (pseudosections du bas) : du sable a été rajouté à l'interface, le milieu comporte un volume plus résistant, et les valeurs supérieures à 1 montrent les points où la flèche a une influence.

Lorsque la ligne n'est enfouie qu'à $4 \mathrm{~cm}$ sous l'interface (figure 5, pseudosections de gauche), l'effet d'une morphologie est surtout visible pour $\mathrm{AB} / 2<0,2 \mathrm{~m}$ : le rayon d'investigation des petits dispositifs AMNB atteint l'interface avec l'eau, et la morphologie est bien localisée. Remarquons cependant que la zone affectée est diffuse et s'étend environ de $\mathrm{x}=0,2$ à $\mathrm{x}=0,4 \mathrm{~m}$, soit une largeur de $20 \mathrm{~cm}$, alors que la largeur moyenne des morphologies est inférieure à $10 \mathrm{~cm}$. Ceci est dû au caractère intégrant de la méthode électrique. Une interprétation quantitative est ensuite indispensable pour décrire la topographie avec une bonne résolution. 

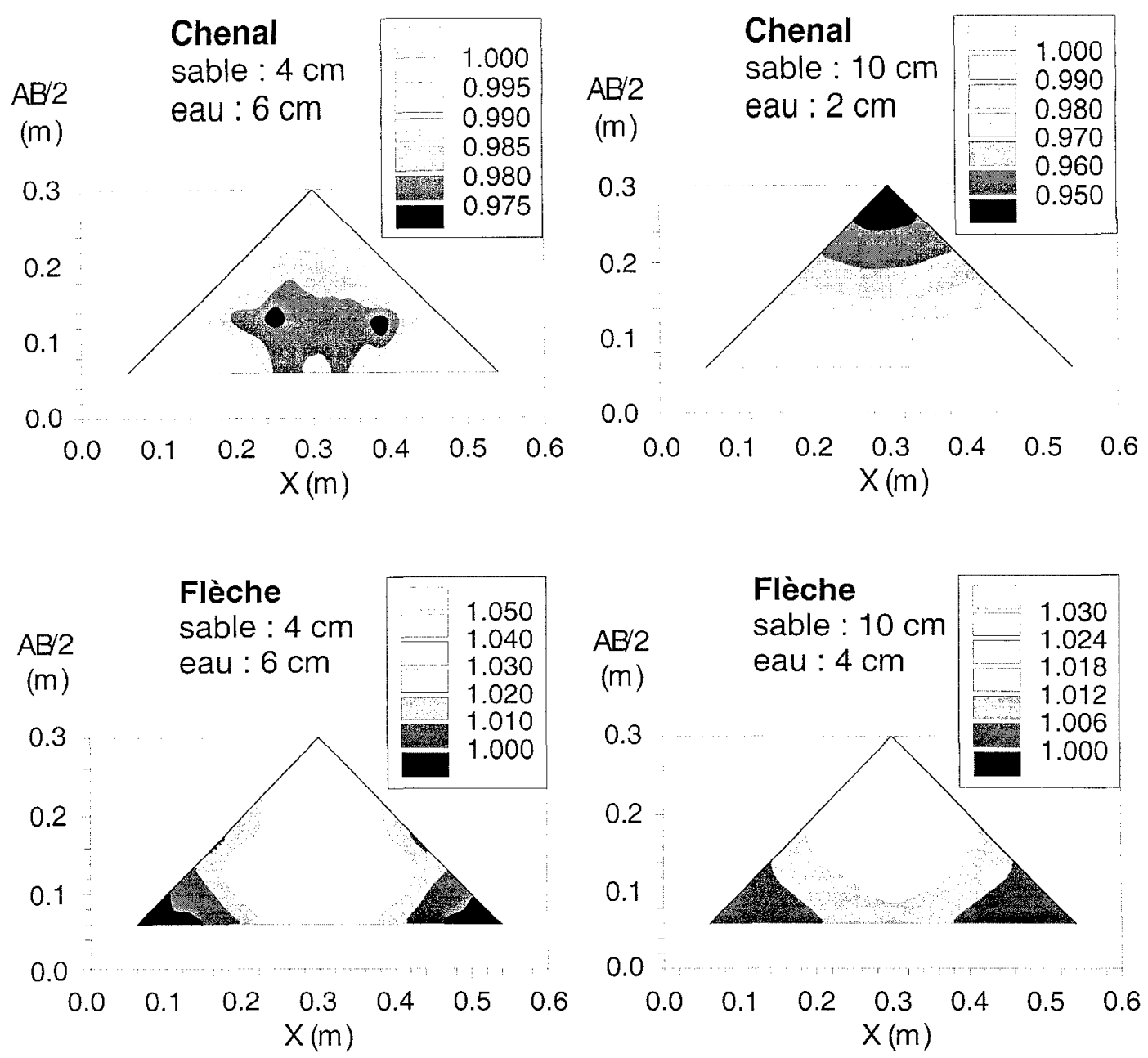

Figure 5. Pseudosections relatives de résistivité apparente. Apparent resistivity relative pseudosections.

Lorsque la ligne est enfouie à $10 \mathrm{~cm}$ sous l'interface (pseudosections de droite), le volume séparant la ligne d'électrodes de l'interface est important (par rapport à l'écartement minimal des électrodes, soit $4 \mathrm{~cm}$ ) et l'effet d'une morphologie n'est perceptible que par les grands dispositifs $(\mathrm{AB} / 2>20 \mathrm{~cm})$, qui ont un volume d'investigation suffisant autour de la ligne. Mais ils sont très intégrants et représentent peu de points de mesure : la résolution spatiale s'en trouve dégradée. Pour interpréter ces résultats de manière quantitative, il faut disposer d'un algorithme d'inversion pouvant partir d'un modèle de répartition des résistivités pour ajuster une topographie de l'interface. La problématique habituelle, en géoélectrique de subsurface, est au contraire que l'on connait la topographie et on cherche à ajuster les résistivités dans le terrain (Loke and Barker, 1996). Les logiciels d'inversion actuellement disponibles (ex. RES2DINV, Loke) ne répondent donc pas bien à notre problème. 
Cependant, l'ensemble des essais effectués et leur comparaison nous fournissent une estimation de la sensibilité de la méthode en eau douce. La limite de détectabilité se situe pour des dimensions de l'ordre de la moitié de celles présentées dans le tableau 4. À l'échelle un, une flèche ou un chenal de hauteur minimale $0,5 \mathrm{~m}$ et de largeur minimale $1,50 \mathrm{~m}$ sera détectable par une ligne comportant une électrode au moins tous les $2 \mathrm{~m}$, enfouie sous $2 \mathrm{~m}$ de sédiments et sous $3 \mathrm{~m}$ d'eau. La résolution sera d'autant meilleure que le niveau d'eau sera faible.

\section{Conclusions}

Les essais réalisés en modèle réduit nous ont permis une première confrontation de la méthode à des conditions expérimentales. Les résultats et leurs tendances, dont la logique est conforme aux comportements électriques attendus, donnent les premières estimations de sensibilité en eau douce. La résolution de la méthode est fortement conditionnée par la hauteur d'eau et par la profondeur d'enfouissement de la ligne, qui devra être minimisée.

La suite de l'étude de faisabilité va consister en modélisations numériques 3D d'une part (validations du modèle réduit, études paramétriques sur des sites réels) et d'autre part en essais in situ, en eau salée.

Enfin, une méthodologie d'interprétation devra être développée, en adaptant une procédure d'inversion de données électriques à la problématique d'ajustement d'une topographie d'interface eau / sédiments, et en tenant compte, le cas échéant, du comportement mécanique des sédiments saturés: par exemple une flèche sableuse, qui fait augmenter les résistivités apparentes mesurées du fait de son volume et son contraste de résistivité avec l'eau, a également un rôle de surcharge sur les sédiments en place, ce qui pourrait modifier leur résistivité.

\section{Références}

[1] Maupin R. (1999) - Validité des techniques de géophysique électrique pour la mesure des évolutions topographiques en environnement littoral. Rapport présenté au CNAM, en vue d'obtenir le titre d'Ingénieur Diplômé par l'État, 72 p.

[2] Lagabrielle R. (1984) - La prospection électrique par courant continu en mer. Bulletin de liaison des Laboratoires des Ponts et Chaussées, ${ }^{\circ} 132,7 \mathrm{p}$.

[3] Baumgartner F. (1996) - A new method for geoelectrical investigations underwater. Geophysical Prospecting, 44, pp.71-98.

[4] Loke M.H. and Barker R. (1996) - Rapid least-squares inversion of apparent resistivity pseudosections by a quasi-Newton method. Geophysical Prospecting, 44, pp.131-152.

\section{Remerciements}

Nous remercions IRIS Instruments pour le prêt de matériel d'acquisition adapté. 